\title{
The calibration of the DSCOVR EPIC multiple visible channel instrument using MODIS and VIIRS as a reference
}

\author{
Conor Haney*a, David Doelling ${ }^{\mathrm{b}}$, Patrick Minnis ${ }^{\mathrm{b}}$, Rajendra Bhatt ${ }^{\mathrm{a}}$, Benjamin Scarino ${ }^{\mathrm{a}}$, \\ Arun Gopalan $^{\mathrm{a}}$ \\ ${ }^{a}$ SSAI, 1 Enterprise Pkwy, Ste 200, Hampton, VA 23666 USA \\ ${ }^{b}$ NASA Langley Research Center, 1 Nasa Dr., Hampton, VA 23666 USA \\ *conor.o.haney@nasa.gov; phone 1.757.951.1671; http://www-pm.larc.nasa.gov
}

\begin{abstract}
The Deep Space Climate Observatory (DSCOVR), launched on 11 February 2015, is a satellite positioned near the Lagrange-1 (L1) point, carrying several instruments that monitor space weather, and Earth-view sensors designed for climate studies. The Earth Polychromatic Imaging Camera (EPIC) onboard DSCOVR continuously views the sunilluminated portion of the Earth with spectral coverage in the UV, VIS, and NIR bands. Although the EPIC instrument does not have any onboard calibration abilities, its constant view of the sunlit Earth disk provides a unique opportunity for simultaneous viewing with several other satellite instruments. This arrangement allows the EPIC sensor to be intercalibrated using other well-characterized satellite instrument reference standards. Two such instruments with onboard calibration are MODIS, flown on Aqua and Terra, and VIIRS, onboard Suomi-NPP.

The MODIS and VIIRS reference calibrations will be transferred to the EPIC instrument using both all-sky ocean and deep convective clouds (DCC) ray-matched EPIC and MODIS/VIIRS radiance pairs. An automated navigation correction routine was developed to more accurately align the EPIC and MODIS/VIIRS granules. The automated navigation correction routine dramatically reduced the uncertainty of the resulting calibration gain based on the EPIC and MODIS/VIIRS radiance pairs. The SCIAMACHY-based spectral band adjustment factors (SBAF) applied to the MODIS/ VIIRS radiances were found to successfully adjust the reference radiances to the spectral response of the specific EPIC channel for over-lapping spectral channels. The SBAF was also found to be effective for the nonoverlapping EPIC channel 10. Lastly, both ray-matching techniques found no discernable trends for EPIC channel 7 over the year of publically released EPIC data.
\end{abstract}

Keywords: DSCOVR, EPIC, Aqua, MODIS, Suomi-NPP, VIIRS, Radiometric Calibration, Deep Convective Clouds (DCC)

\section{INTRODUCTION}

The Deep Space Climate Observatory (DSCOVR) spacecraft is a satellite developed with the intent to monitor space weather and provide other climate-related operations pertaining to the Earth. Launched on 11 February 2015, DSCOVR orbits the Sun near the first Lagrangian point (L1), located approximately 1.5 million kilometers from Earth between the Earth and the Sun. Being located at L1 allows DSCOVR to have a continuous view of the both the Sun and the sunlit disk of Earth, providing unique research opportunities for studying and monitoring both bodies. In addition to the Sun-facing instruments onboard DSCOVR, it also has two Earth-facing instruments: The National Institute of Standards and Technology Advanced Radiometer (NISTAR) and the Earth Polychromatic Imaging Camera (EPIC). NISTAR was designed to measure the Earth's radiation budget, measured across four channels. These channels measure total outgoing flux $(0.2-100 \mu \mathrm{m})$, RSB $(0.2-4 \mu \mathrm{m})$, NIR $(0.7-4 \mu \mathrm{m})$, and VIS $(0.3-1 \mu \mathrm{m})$. EPIC, the focus of this study, was designed to monitor aerosols, ozone, clouds, and vegetative properties, using 10 channels that span the ultraviolet (UV), visible (VIS), and near-infrared (NIR) spectrums. 
It is important for any scientific instrument to be properly calibrated in order to ensure the full potential of the data. Unfortunately, DSCOVR EPIC does not have an onboard calibration system. Instead, it has other means of providing a calibration, such as monthly dark current measurements while EPIC's shutters are closed. Being a CCD array, flatfielding was initially performed for EPIC during ground characterization. However, this is not feasible while on orbit. The filter wheels that determine the EPIC channels are also radiometrically calibrated against the moon for stability monitoring.

The DSCOVR earth view sensors always face the sunlit-disk of Earth, thus providing a unique opportunity for inter-calibration of the EPIC channels by using well-calibrated instruments aboard various low-Earth orbit (LEO) satellites. In particular, the Moderate Resolution Imaging Spectroradiometer (MODIS) instrument onboard Aqua, and the Visible Infrared Imaging Radiometer Suite (VIIRS) instrument onboard Suomi-NPP, each have several channels that could be used as references for inter-calibration with the EPIC channels, with the exception of EPIC's UV channels (bands 1 through 4). The Aqua-MODIS Collection 6 visible channel calibration stability was found to be within $1 \%{ }^{l}$. The NPP-VIIRS Land (Product Evaluation and Analysis Tool Element) PEATE L1B calibration stability was found to be $1 \%{ }^{2}$. The inter-calibration will allow for accurate monitoring of the EPIC instrument's performance and any possible temporal degradation. This study aims to assess the navigation, the feasibility of inter-calibrating EPIC with MODIS and VIIRS utilizing ray-matching over all-sky ocean and deep convective cloud conditions, and to determine the temporal degradation of the EPIC instrument channels.

For this study, many of the ray-matching techniques that the CERES project employs to calibrate geostationary radiances, which are used to estimate diurnal fluxes between CERES observed fluxes, are applied to EPIC ${ }^{345}$. The allsky ocean and DCC ray-matching validation techniques follow the approach of Doelling et al. $2016^{6}$, which were used to validate the CERES geostationary calibration methodology.

\section{DATA}

There are three different versions of this EPIC level 1B data used for this study. The NASA Goddard Space Flight Center (GSFC) publicly released its first version of the EPIC L1B v1 data product (https://earthdata.nasa.gov/search?q=DSCOVR) to the public on 20 July 2016. The current EPIC record begins on 13 June 2015. This version is denoted as version 01. In addition to this, two unreleased datasets for 5 September 2015 were used for this study. The first dataset has an updated navigation algorithm, and the second has both the updated navigation and stray-light correction applied. These two datasets were made available to the DSCOVR science team.

Each EPIC channel has a set of associated parameters describing the geo-location, viewing geometry, and solar geometry. The EPIC channels are not co-registered because EPIC uses a rotating filter wheel to measure each channel using the same CCD array of detectors. Typically, a few seconds separates channel images. EPIC operations usually sample the Earth about every hour. The EPIC image of 2048 by 2048 pixels encompasses the entire Earth, with a nominal pixel resolution of $8 \times 8 \mathrm{~km}^{2}$, which have an optical resolution of $12 \times 12 \mathrm{~km}{ }^{2}$ (http://epic.gsfc.nasa.gov/epic.html). The EPIC instrument measures radiance in terms of counts per second.

Aqua-MODIS Collection 6 L1B data are used in this study. The 1-km nominal pixel resolution MODIS data have been sub-sampled at 2-km. The NPP-VIIRS data are from the Land PEATE L1B version 03110 product. The Land PEATE product has been reprocessed using the IDPS software with consistent calibration over the record in order to remove any known temporal VIIRS calibration anomalies that may exist in the VIIRS IDPS product. The VIIRS bands have two pixel resolutions. The M-bands have a nominal pixel resolution of $750 \mathrm{~m}$, and the I bands have a pixel resolution of $375 \mathrm{~m}$. Unlike MODIS, VIIRS employs pixel aggregation to maintain the pixel resolution over the crosstrack scan. For this study the I1 band has been subsetted to the $750-\mathrm{km}$ resolution dataset.

The EPIC channel data will be inter-calibrated using either MODIS or VIIRS as a calibration reference. Each MODIS and VIIRS band is independently calibrated based on solar diffuser observations. Calibration differences between MODIS and VIIRS have not been removed for this study. Therefore, EPIC calibration based on MODIS and VIIRS may differ due to the onboard calibration differences between MODIS and VIIRS, as well due to methodology. To avoid confusion and excessive verbiage, the instrument bands will be denoted as listed in Table 1 . EPIC bands 1 
through 4 are in the UV spectrum and do not have relevant MODIS or VIIRS channel counterparts, and are therefore excluded from this analysis.

Table 1. Instrument channel identifiers and their corresponding wavelengths.

\begin{tabular}{|c|c|c|c|c|c|}
\hline \multicolumn{2}{|c|}{ EPIC } & \multicolumn{2}{c|}{ MODIS } & \multicolumn{2}{c|}{ VIIRS } \\
\hline Band ID & Wavelength (nm) & Band ID & Wavelength (nm) & Band ID & Wavelength (nm) \\
\hline E5 & 443 & A1 & 645 & M3 & 488 \\
\hline E6 & 551 & A2 & 865 & M4 & 555 \\
\hline E7 & 680 & A3 & 470 & M5 & 672 \\
\hline E8 & 688 & A4 & 555 & I1 & 640 \\
\hline E9 & 764 & & & M7 & 865 \\
\hline E10 & 780 & & & \multicolumn{2}{|l}{} \\
\cline { 1 - 1 } & & &
\end{tabular}

\section{METHODOLOGY}

\subsection{Navigation Correction}

An initial inspection of the individual channel EPIC images revealed, that they were not properly navigated. The inter-calibration between two sensors, using either simultaneous nadir overpass (SNO) or ray-matched radiance pairs, requires both coincident and collocated imagery. Because the EPIC and LEO images do not have the same satellite projection, the data are averaged on a $0.25^{\circ}$ latitude by longitude grid. In order to utilize the near-nadir high spatial resolution portion of the EPIC imagery, only MODIS or VIIRS granules that encompass $\pm 30^{\circ}$ in latitude are collocated with EPIC. The EPIC and LEO images are also matched within 15 minutes in time. The $0.25^{\circ}$ grid resolution should mitigate any advection-induced spatial discrepancies that may occur within 15 minutes. The navigation quality of each gridded image can easily be verified by looking for a visible contrast between clear-sky ocean and land. Fig. 1a displays the E7 image taken on 5 September 2015 at 8:36 GMT. Fig. 1b shows the corresponding (VIIRS) I1 8:35 GMT granule. Clearly, the E7 image is not aligned with the west coast of India, whereas the well-navigated I1 granule is. Note that the spatial distribution of the cloud and surface reflectances are very similar between images, although may differ due to viewing geometry differences. The sun-glint feature (the vertical bright stripe over clear-sky ocean) in the I1 image does not appear in the E7 image. Given the size of the EPIC image compared to the size of the LEO granule, there will often be multiple consecutive LEO granules for a particular EPIC image that meet this temporal criteria. When a single E7 full disc image was evaluated with several contiguous I1 granules, it was found that the E7 navigation shift was not uniform across the image. Therefor the EPIC image must be spatially adjusted for each collocated VIIRS granule.

Because visually inspecting the EPIC navigation adjustments is feasibly impossible, an automated image alignment technique was developed based on the EPIC and LEO $0.25^{\circ}$ resolution grids. The EPIC grid domain is extended by $5^{\circ}$ in latitude and longitude of the collocated LEO granule boundary to allow the EPIC image to be shifted to best match the collocated LEO granule. Only grid locations or inter-calibration footprints (ICF) with both an E7 and LEO (A1 or I1) valid radiance pair are linearly regressed, and the coefficient of determination $\left(\mathrm{R}^{2}\right)$ is computed. Next, the EPIC grid is incrementally shifted from -5 to +5 ICFs in both the latitudinal and longitudinal directions, incrementing by one ICF at a time. Of the 121 grid locations tested, the grid location with the greatest $R^{2}$ should be the most aligned or best navigated EPIC image with respect to the collocated LEO image. Fig. 1c demonstrates the accuracy of the navigation correction, where the EPIC image is now aligned with the west coast of India as well as the cloud features. Fig. 1d shows the $\mathrm{R}^{2}$ field for this particular image shift, where a latitude offset of +1 (North) and an longitude offset of +2 (East) contains the highest $\mathrm{R}^{2}$, and was subsequently used for the navigation correction. This navigation technique works best were there are many contrasting features such as clouds and coastlines, which is typical over the tropics. However this technique is not suited over polar ice caps with few contrasting features. 

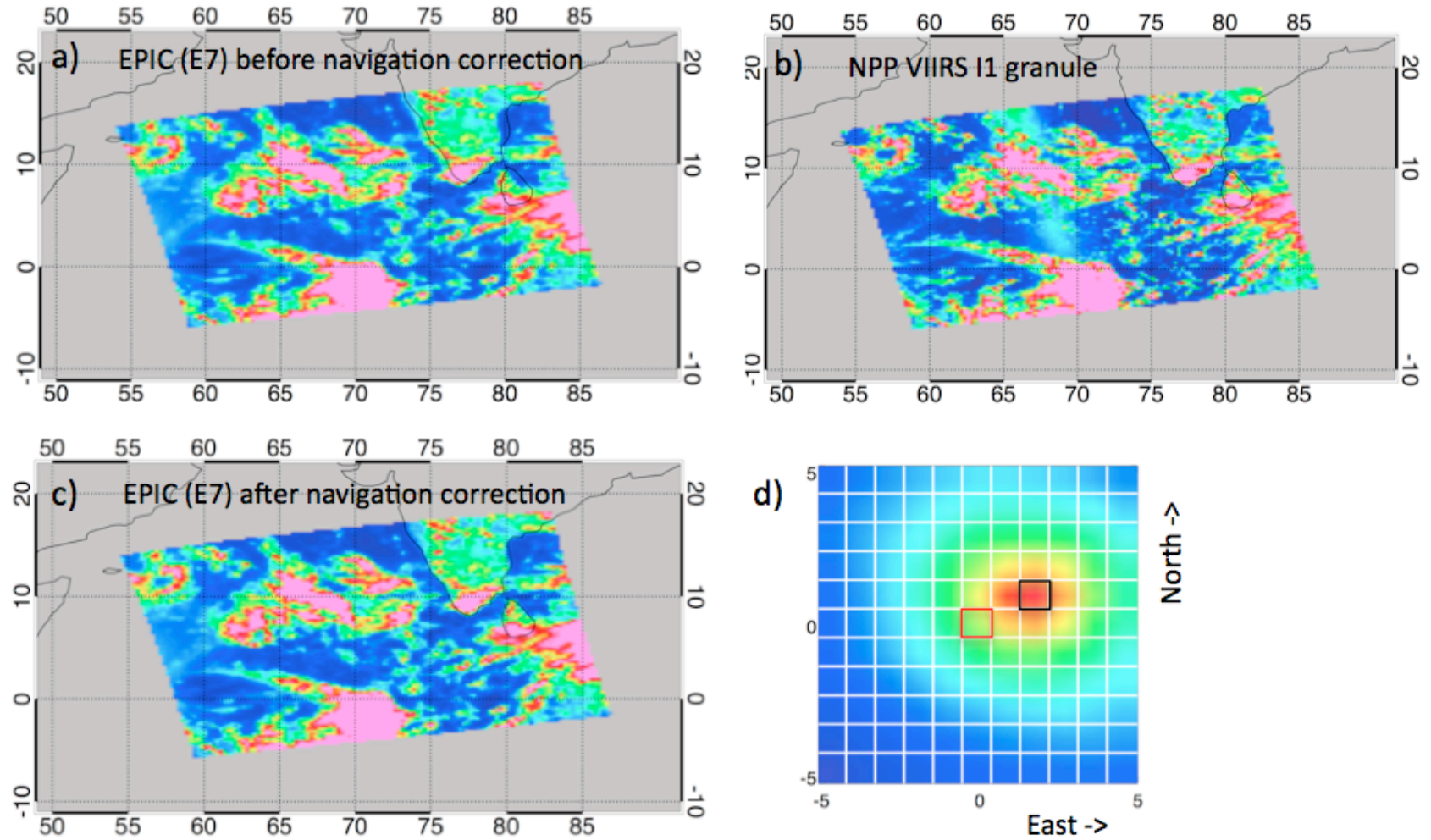

Figure 1. a) EPIC band 7 gridded image corresponding to the NPP-VIIRS I1-channel granule domain, with no navigation correction applied for 5 September 2015, 8:36 GMT. b) The NPP-VIIRS I1-channel 8:35 GMT granule corresponding to $a$. c) Same as $a$, but with the navigation correction applied. d) The EPIC $\pm 5 \mathrm{R}^{2}$ field corresponding to a). The red box contains the original navigation $R^{2}$, where the black box contains the optimized navigation shift (1 North, 2 East) $R^{2}$. The blue represents $R^{2}$ close to 0 and the red close to 1 . The y-axis corresponds to the latitude shift (positive North), and the $\mathrm{x}$-axis corresponds to the longitude shift (positive East).

\subsection{Gridded Ray-matching}

Once the navigation correction in Section 2.1 is applied, instantaneous coincident collocated angle matched EPIC and LEO pair radiances, referred to as ray-matching in this study, are used to compute the EPIC channel gain based on the given LEO reference sensor. Unlike the navigation correction, which simply constitutes a match in time and location, ray-matching also matches the viewing and solar geometries of the sensor. This study follows closely the raymatching technique used by Morstad et. al. $2011^{5}$ and Doelling et al. $2013^{7}$. First the EPIC and LEO radiance are aggregated onto a $0.5^{\circ}$ latitude by longitude grid to further reduce the impact of the EPIC navigation error and advection-induced spatial shifts caused by time difference. The radiance pairs are then filtered to angle-match the data. The EPIC and LEO viewing zenith angle (VZA), relative azimuth angle (RZA), and scattering angle difference must be within $15^{\circ}$. The overall VZA is limited to $40^{\circ}$ and the solar zenith angle (SZA) is limited to $84.26^{\circ}(0.1$ cosine SZA). In order to avoid large spectral band differences, land regions are not used.

In order to obtain more homogeneous reflectances within the $0.5^{\circ}$ region, a spatial visible sigma (SVS) threshold is applied to the gridded ray-matching data. The SVS is the instantaneous $0.5^{\circ}$ regional standard deviation divided by the mean radiance. The smaller the SVS the more uniform the region. SVS is unique to each channel pair and is empirically derived. For gridded ray-matching, the SVS value is 0.1 for E5/A3 and E6/A4, while SVS is 0.2 for E7/A1.

Ideally, ray-matching utilizes precisely matched radiances, however this is rarely observed. Some angular tolerance is required in order to obtain sufficient sampling across the sensor dynamic range. Too much tolerance, however, may introduce a bias in the ray-matched gain, especially for anisotropic scenes. Therefore, a graduated angle matching (GAM) filter is applied, which varies the angular tolerance from the clear-sky ocean scenes to bright cloud 
conditions. The clear-sky conditions over the ocean are the most anisotropic and therefore require the strictest angle matching, whereas bright clouds are more Lambertian, which allows for a greater angular tolerance. This technique requires that all matched pairs with a (reference) radiance less than $100 \mathrm{Wm}^{-2} \mathrm{sr}^{-1} \mu \mathrm{m}^{-1}$ must have a $\Delta \mathrm{VZA}<5^{\circ}$ and $\triangle \mathrm{RZA}<5^{\circ}$. For (reference) radiances between $100 \mathrm{Wm}^{-2} \mathrm{sr}^{-1} \mu \mathrm{m}^{-1}$ and $200 \mathrm{Wm}^{-2} \mathrm{sr}^{-1} \mu \mathrm{m}^{-1}$, a $\Delta \mathrm{VZA}<10$ and $\Delta \mathrm{RZA}<10$ tolerance is applied. For radiances greater than $200 \mathrm{Wm}^{-2} \mathrm{sr}^{-1} \mu \mathrm{m}^{-1}$, the nominal angular differences are maintained. GAM is only applied if sampling is sufficient for dark radiances, thereby maintaining the dynamic range.

The EPIC radiances are then normalized to the SZA of the LEO sensor by applying a cosine of the solar zenith angle ratio. A spectral band adjustment factor, as outlined in Section 2.3, is then applied to account for the EPIC and LEO band spectral response function (SRF) differences. Finally, the 15-minute time matched collocated ray-matched EPIC and LEO radiance pairs are linearly regressed. A least squares linear (minimize $\mathrm{x}$ distance to line) regression (linear fit), a linear fit through the offset (force fit), a principal component (least distance to the line) regression (PC fit), and a reversed axis least squares (minimize y distance to line) fit (SLPyx) is performed. The agreement between the force and linear fit can then be used to quantify the navigation improvements.

Fig. $2 a$ and $2 b$ show the E7 and I1 ray-matched radiance pairs and the corresponding fits for the EPIC and VIIRS images shown in Fig. 1. The standard error is based on the linear fit. The standard error (SE) for nominal navigation is $48 \%$, whereas after applying the navigation correction procedure in Section 2.1, the SE has been reduced to $12 \%$, thus validating the accuracy of the navigation correction. The $\mathrm{x}$-axis offset based on the PC fit has been reduced from -3978 to 199 , and the offset is now much closer to zero. Also, all of the fits are now in much closer agreement. The force and linear fit agreement before and after navigation correction are $14.3 \%$ and $0.7 \%$, respectively, with $0 \%$ representing full agreement.
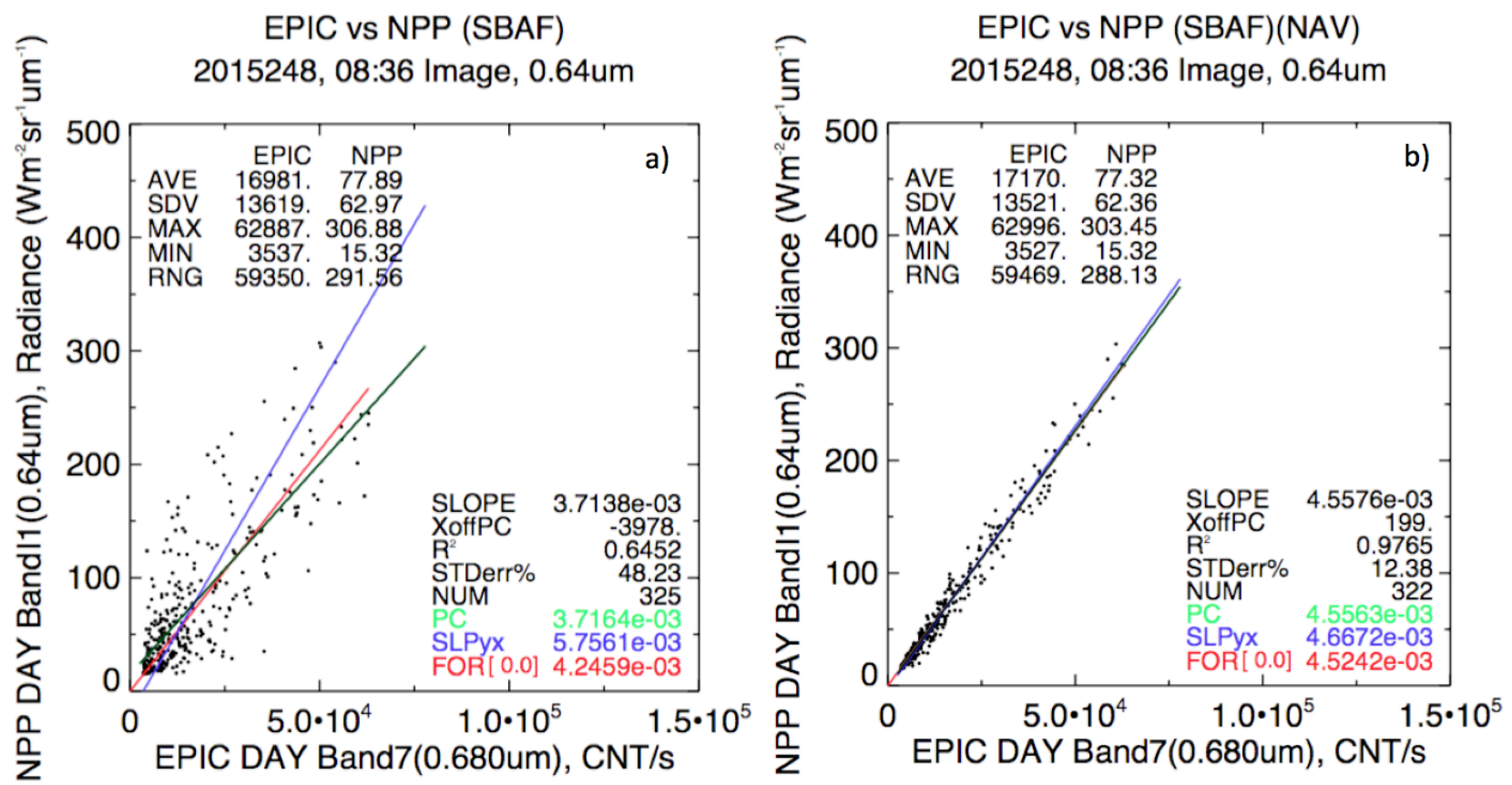

Figure 2. a) The ray-matched radiance E7/I1 radiance pair scatterplot for the image in Fig. 1, without the navigation correction applied. b) Same as $a$, except with the navigation correction applied. SLOPE refers to the linear fit gain, XoffPC is the PC fit X-axis offset, $\mathrm{R}^{2}$ is the linear fit coefficient of determination, STDerr\% is the linear fit standard error in \%, NUM is the number radiance pairs, PC is the principal component fit gain, SLPyx is the reversed axis linear fit gain, and FOR is the force fit gain, where the number in the parenthesis is the associated offset ( 0 in this case). 


\subsection{SBAF Correction}

In order to account for the SRF differences between EPIC and MODIS/VIIRS bands, a spectral band adjustment factor (SBAF) is applied to the LEO band radiance as if the LEO channel had the corresponding EPIC band SRF. The SBAF is based on ENVISAT's Scanning Imaging Absorption Spectrometer for Atmospheric Chartography (SCIAMACHY) hyper-spectral radiances that have been convolved with the EPIC and LEO SRFs. A radiance pair regression is performed using SCIAMACHY footprints based on the same underlying Earth conditions used with the EPIC and LEO ray-matching algorithm. For instance, the gridded ray-matching in Section 2.2 is performed over an allsky tropical ocean Earth scene condition, and thus the SBAF is derived from SCIAMACHY data retrieved over all-sky tropical ocean regions. The SBAF regression can easily be obtained from the NASA LaRC SBAF Web-tool (http://cloudsgate2.larc.nasa.gov/SBAF), which takes SCIAMACHY footprints for a specified scene type and convolves its spectra with the SRFs of a reference and target instrument channel to form a pair of pseudo radiances, which are then regressed to a user-defined polynomial order to yield the $\mathrm{SBAF}^{8}$.

The application of the SCIAMACHY-based SBAF tool for Fig. 2 is shown in Fig. 3. Fig. 3a shows the SRFs for E7 and I1. Note that the EPIC SRF has a 2-nm (FWHM) bandwidth, whereas the I1 has a 20-nm bandwidth. Over this wavelength range the SCIAMACHY spectral resolution is $0.48 \mathrm{~nm}$, which should be sufficient for estimating the SBAF associated with these two bands. Fig. 3b shows the corresponding NASA LaRC SBAF web-tool generated plot. A $2^{\text {nd }}$-order regression was chosen to best represent the SBAF for the two bands over an all-sky tropical ocean domain. The lowest regression is chosen, after which applying higher order regression fits no longer reduce the SE of the fit. The force, linear, $2^{\text {nd }}$-order and $3^{\text {rd }}$-order fits had standard errors of $1.903 \%, 1.408 \%, 1.215 \%$ and $1.210 \%$, indicating no significant reduction in SBAF uncertainty for regression orders greater than two. As determined from Fig. $3 \mathrm{~b}$, not accounting for SBAF could cause a 5\% gain difference between E7 and I1. Note that the SBAF is very dependent on the underlying Earth reflected spectra, thus special attention must be made to matching the calibration target and scene conditions when applying an SBAF.
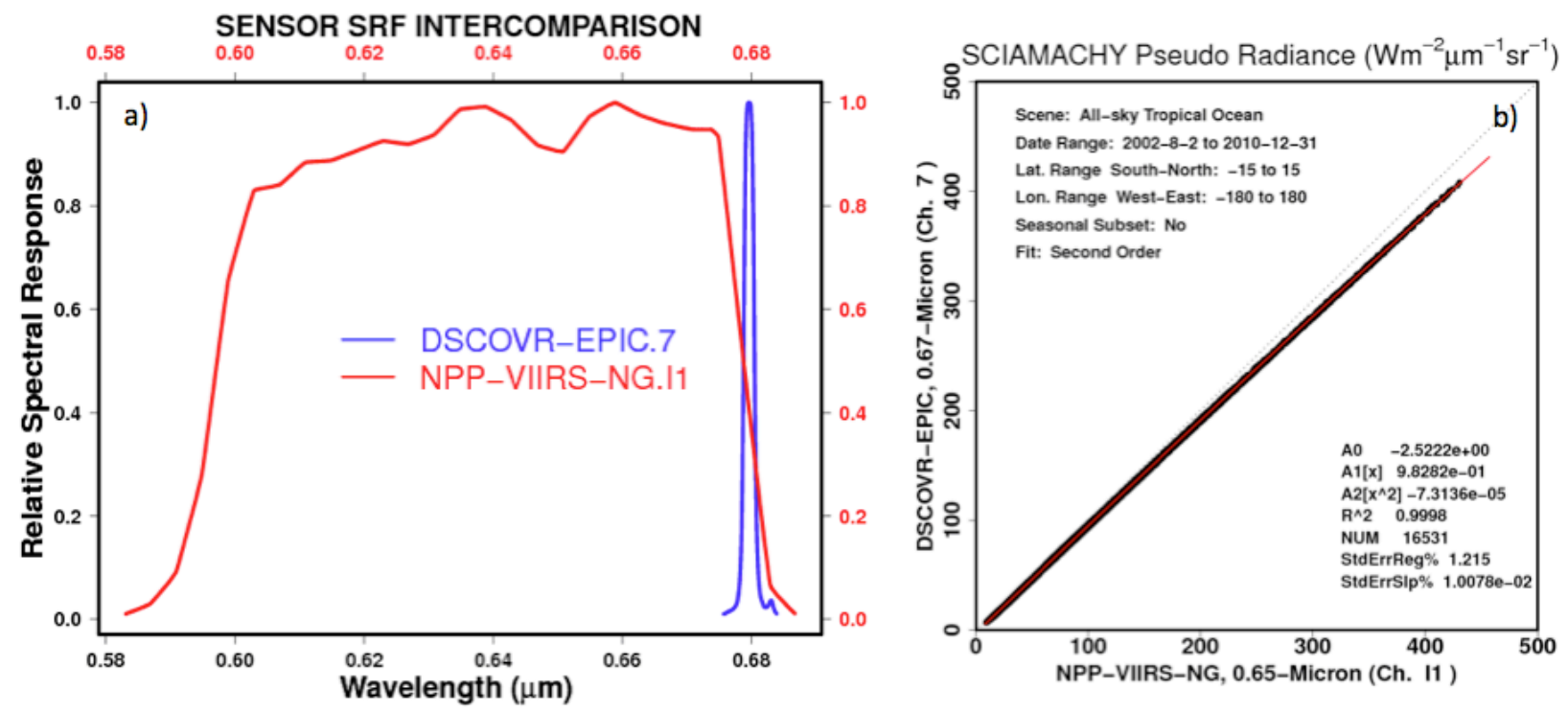

Figure 3. a) The normalized spectral response functions for E7 and I1. b) The NASA LaRC SCIAMACHY-based SBAF web-tool output plot generated for E7/I1 over all-sky tropical ocean domain.

\subsection{Ray-matching}

As noted in Section 2.3, the SBAF SE, or uncertainty, is dependent on the underlying Earth reflected spectra. Deep Convective Clouds (DCC) are the brightest, most isotropic Earth targets, with a nearly flat spectral response over visible wavelengths ${ }^{9}$. They are also located at the tropopause and therefore are not impacted by water vapor absorption in the NIR ${ }^{10}$. Therefore, for wavelengths less than $1 \mu \mathrm{m}$, DCC have the smallest SBAF uncertainty of all Earth targets. The same NASA LaRC SBAF web-tool is used to determine the DCC SBAF (not shown). The E7/I1 SBAF force fit was 
0.947, and the associated SE was $0.55 \%$, almost $1 / 3$ less than the all-sky tropical ocean domain SBAF SE (Fig. 3b). This suggests that the SBAF for DCC is very linear. However, the DCC frequency over the tropics is on order of $0.5 \%{ }^{11}$. In order for DCC ray-matching to be successful, sufficient DCC sampling is required. Because DCC are found across the entirety of the tropics, unlike desert targets, and because the EPIC images always encompass the sunlit portion of the Earth, sufficient DCC sampling is easily achievable.

A similar DCC ray-matching algorithm as that described in Doelling et al. 2016 is followed ${ }^{6}$. The DCC are identified using an 11- $\mu \mathrm{m}$ brightness temperature (BT) threshold. The EPIC sensor does not have an IR sensor because Earth emitted IR radiance are too weak to be measured at L1 using Earth-viewing IR detectors. Even if an IR sensor were available, it would not be co-registered with the visible channels due to the filter wheel design of the insrtrument. For DCC ray-matching, the MODIS B31 or VIIRS M15 band BTs are used. Beause the EPIC and LEO images were navigated using a grid resolution of $0.25^{\circ}$, the EPIC DCC algorithm uses the same $0.25^{\circ}$ resolution navigation-corrected dataset. This prerequisite implies that only DCC cells that have an extent of $\sim 30 \mathrm{~km}$ are sampled. The DCC grid locations are identified using a BT threshold of less than $220 \mathrm{~K}$. Additionally, only DCC ray-match pairs with an EPIC and LEO $\triangle \mathrm{VZA}<15^{\circ}$, a $\triangle \mathrm{RZA}<25^{\circ}$, a $\triangle \mathrm{SZA}<5^{\circ}$, and a scattering angle difference $<15^{\circ}$ are accepted. In order to take advantage of the more Lambertian part of the DCC reflectance, both EPIC and LEO VZA are restricted to $<40^{\circ}$, and SZA $<40^{\circ 9}$. The DCC ray-matching also utilizes a spatial IR homogeneity threshold (SIR), which is the $0.25^{\circ}$ regional BT spatial standard deviation. For DCC, the E7/I1 SVS equals 0.05 , and the SIR is $2.5 \mathrm{~K}$. The spatial homogeneity thresholds attempt to isolate the DCC cores from the anvils.

The EPIC radiances are then normalized to the SZA of the LEO sensor by applying a cosine of the solar zenith angle ratio. Then the DCC SBAF is applied to the LEO radiances. Similarly, the EPIC and LEO DCC ray-matched radiance pairs are regressed to obtain the EPIC calibration gain. Both the gridded all-sky ocean and DCC ray-matched gains should be equal. Agreement among multiple independent calibration validates all methods.

\section{RESULTS}

\subsection{Stray-light Correction}

In order to test the quality of the stray-light corrected version of the EPIC data, both the non-stray-light and stray-light-corrected data are inter-calibrated with MODIS using the gridded ray-matching technique described in Section 2.2. The data was made available for the 5 September 2015 case, which was released to the science team. For this comparison, GAM was not applied. SVS, however, was applied.

Figure $4 \mathrm{a}$ shows the gridded ray-matching plots for the non-corrected stray-light dataset, and Fig. $4 \mathrm{~b}$ shows the corrected stray-light dataset for E7/A1. While the regression standard error was reduced slightly, the PC fit offset was reduced from 1301 to 6 , bringing it much closer to the predicted offset value of zero. The gain difference between the force and linear fit was reduced from $3.5 \%$ to $0.1 \%$. The removal of stray light from the optics also lowered the force fit calibration gain by $6.4 \%$. Table 2 provides the E5/A3, E6/A4, E7/A1, and E8/A1 channel pair statistics. All channel pairs reveal a slight decrease in the SE of the linear fit, a significant reduction of the PC fit offset, which is much closer to zero, and linear and force fit gains that are more consistent. Lastly, the stray light correction reduced the calibration gains for all channel pairs. These consistent statistics verify the improvement of the EPIC stray-light correction software applied on 5 September 2015. 

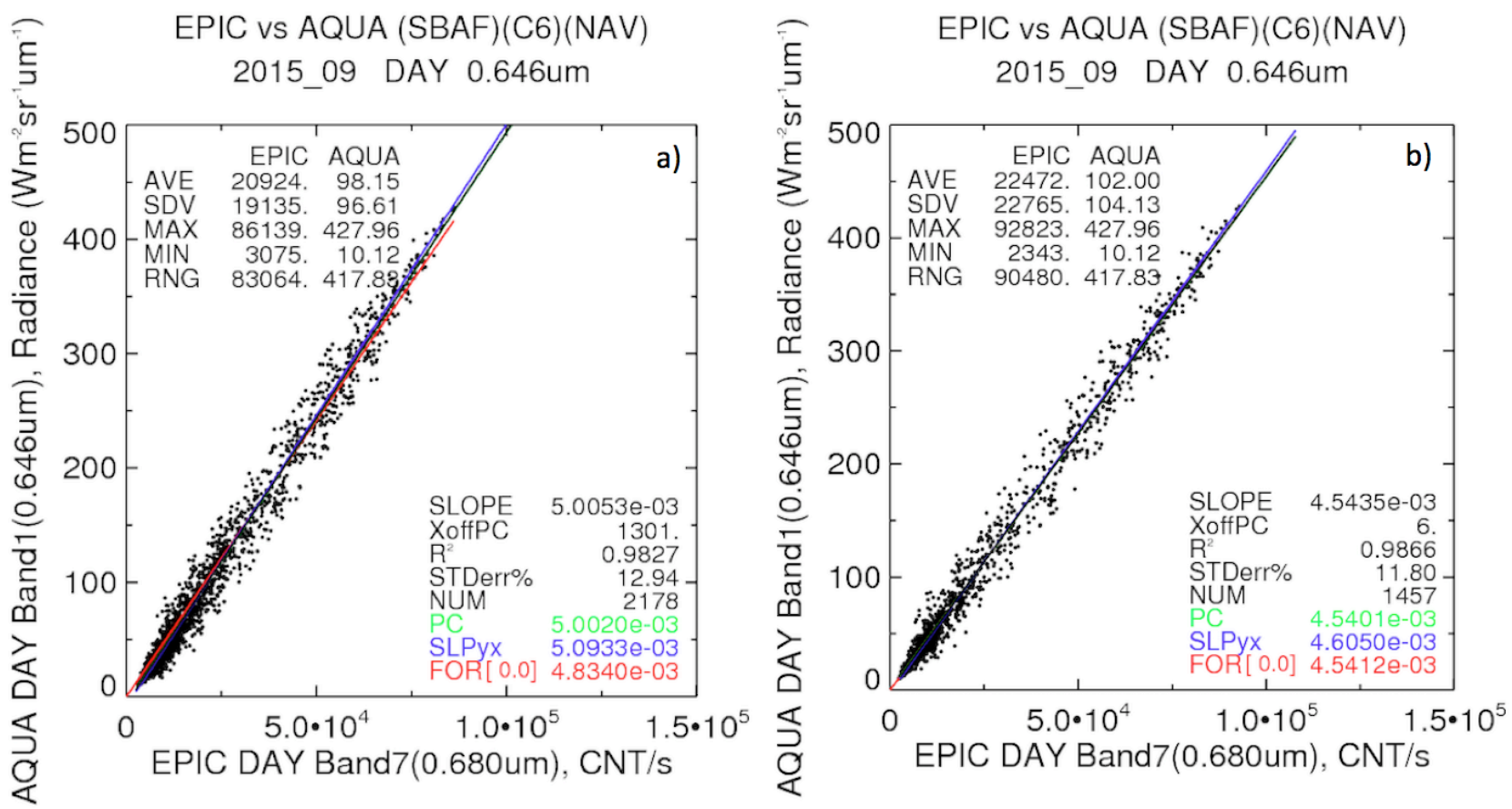

Figure 4. a) The E7/A1 radiance pair plot for 5 September 2015 of the non-stray-light-corrected data. b) The same as $a$, but using the stray-light corrected data. See Fig. 2 for an explanation of the plot statistics.

Table 2. Without stray-light (W/O Stray) and stray-light (Stray) correction statistics based on the E5/A3, E6/A4, E7/A1, E8/A1 channel pair regressions for 5 September 2015.

\begin{tabular}{|c|c|c|c|c|c|c|c|}
\hline & \multicolumn{2}{|c|}{ STDerr\% linear fit } & \multicolumn{2}{c|}{ Linear/force fit } & \multicolumn{2}{c|}{ PC fit X offset } & W/O Stray - Stray \\
\hline Band Pairing & W/O Stray & Stray & W/O Stray & Stray & W/O Stray & Stray & Gain Difference \\
\hline E5/A3 & 7.8 & 8.0 & $+4.1 \%$ & $+1.9 \%$ & 1962 & 965 & $-2.6 \%$ \\
\hline E6/A4 & 11.2 & 10.5 & $+2.5 \%$ & $+0.4 \%$ & 1339 & 287 & $-2.4 \%$ \\
\hline E7/A1 & 12.9 & 11.8 & $+3.5 \%$ & $+0.1 \%$ & 2178 & 6 & $-6.4 \%$ \\
\hline E8/A1 & 15.2 & 14.7 & $+1.7 \%$ & $-0.7 \%$ & 2170 & -116 & $-5.6 \%$ \\
\hline
\end{tabular}

\subsection{SBAF Correction}

The same gridded ray-matching statistics can also be used to validate the SBAF correction. In this case, the EPIC channels have a very narrow spectral width and are within $3 \mathrm{~nm}$, whereas the MODIS and VIIRS channels are within $20 \mathrm{~nm}$ for most channels. Table 3 reveals the standard errors of the linear fit and the PC fit X-axis offset for the various EPIC and VIIRS channel pairings. The SBAF is a $2^{\text {nd }}$-order function that should not impact the overall noise of the radiance pairs. The standard errors for most channels increased slightly. However, the PC offset was significantly reduced and much closer to zero than that without applying SBAF. This suggests that the SBAF adjusted the clear-sky radiances more than the bright cloud radiances because bright clouds are more spectrally flat than clear-sky ocean conditions.

While many of the EPIC channels have MODIS and VIIRS channel counterparts, the E9 and E10 channels do not. These channels were designed to determine cloud height using the Oxygen-A absorption band (E9) with its reference window band (E10). This scenario provides an opportunity to test the SCIAMACHY-based SBAF tool (section 2.3) ability to infer the SBAF for non-overlapping spectral channels. The E9 $(0.764 \mu \mathrm{m})$ and E10 $(0.780 \mu \mathrm{m})$ bands can be inter-calibrated with the MODIS A1 $(0.65 \mu \mathrm{m})$ and A2 $(0.86 \mu \mathrm{m})$ bands. The A2 band saturates for large radiance values (e.g., DCC). E9 and E10 can also be inter-calibrated with the VIIRS I1 (640 nm), M5 (672 nm), and M7 $(865 \mathrm{~nm})$ bands. None of the VIIRS band saturate over bright scenes. In this study, the E10 band is inter-calibrated with the VIIRS I1, M5, M7 bands. The E9 absorptive band is the more challenging case and will be addressed in the future. 
Figure 5a shows the E10/I1 radiance pairs and statistics before application of the SBAF. Figure 5b shows the E10/I1 SCIAMACHY footprint pseudo radiance pairs, which have a definite $2^{\text {nd }}$-order distribution, and for which the standard error about the fit is $2.95 \%$. The standard error is larger than that for overlapping bands. However, the SBAF standard error is much less than the standard error based on the E10/I1 ray-matched radiance pairs. Figure 5c illustrates the E10/I1 radiance pairs and statistics after application of the SBAF. The PC offset is much closer to zero, thus validating that the SBAF approach is also possible for non-overlapping spectral channels. Also, the linear and force fit gain difference was reduced from $3.3 \%$ to $0.9 \%$ after applying the SBAF. If the spectral band differences were not applied, the force fit calibration gain would be biased by $+1.1 \%$, thus demonstrating the importance of applying SBAF. In fact, Table 3 implies, for EPIC and VIIRS channel pairings, that the linear regression and the force fit are more similar, and therefore validates the use of the SBAF for this inter-calibration study.

If the VIIRS I1, M5, and M7 were calibrated perfectly or to the same radiometric scale, then the SBAF should provide similar calibration gains for E10/I1, E10/M5, and E10/M7. Figure 6 shows the ray-matched radiance pairs for the E10/I1, E10/M5, and E10/M7 with the corresponding statistics. The force fit E10 calibration gains for E10/I1, $\mathrm{E} 10 / \mathrm{M} 5$, and E10/M7 are 5.1839e-3, 5.2621e-3, and 5.2156e-3, respectively. All of the gains are within $1.5 \%$. Interesting enough the greatest difference is between E10/I1 and E10/M5. The force fit E7 calibration gains were compared between E7/I1 and E7/M5, and were found to be 4.4791e-3 and 4.5461e-3, respectively (not shown). For E7 the I1 and M5 calibration gain difference was also found to be $1.5 \%$, with the I1 calibration gain less than the M5 calibration gain. This suggests that the E10/I1 and E10/M5 calibration gain differences are probably due to the I1 and M5 absolute calibration difference, and that the SBAF has successfully taken into account the spectral band differences.

Table 3. Regression standard error and x-offset results for EPIC/VIIRS SBAF analysis.

\begin{tabular}{|c|c|c|c|c|}
\hline & \multicolumn{2}{|c|}{ STDerr\% } & \multicolumn{2}{c|}{ X-Offset } \\
\hline Band Pairing & Without SBAF & SBAF & Without SBAF & SBAF \\
\hline E5/M3 & 11.2 & 9.8 & 4626 & 838 \\
\hline E6/M4 & 13.1 & 13.8 & 109 & -291 \\
\hline E7/I1 & 15.1 & 16.3 & -187 & 13 \\
\hline E7/M5 & 15.5 & 16.4 & 262 & 119 \\
\hline E8/I1 & 16.0 & 18.1 & -440 & -196 \\
\hline E8/M5 & 16.7 & 18.4 & -282 & -167 \\
\hline E10/I1 & 16.2 & 17.3 & -572 & 140 \\
\hline E10/M5 & 16.6 & 17.4 & -273 & 193 \\
\hline E10/M7 & 18.4 & 18.0 & 622 & 439 \\
\hline
\end{tabular}
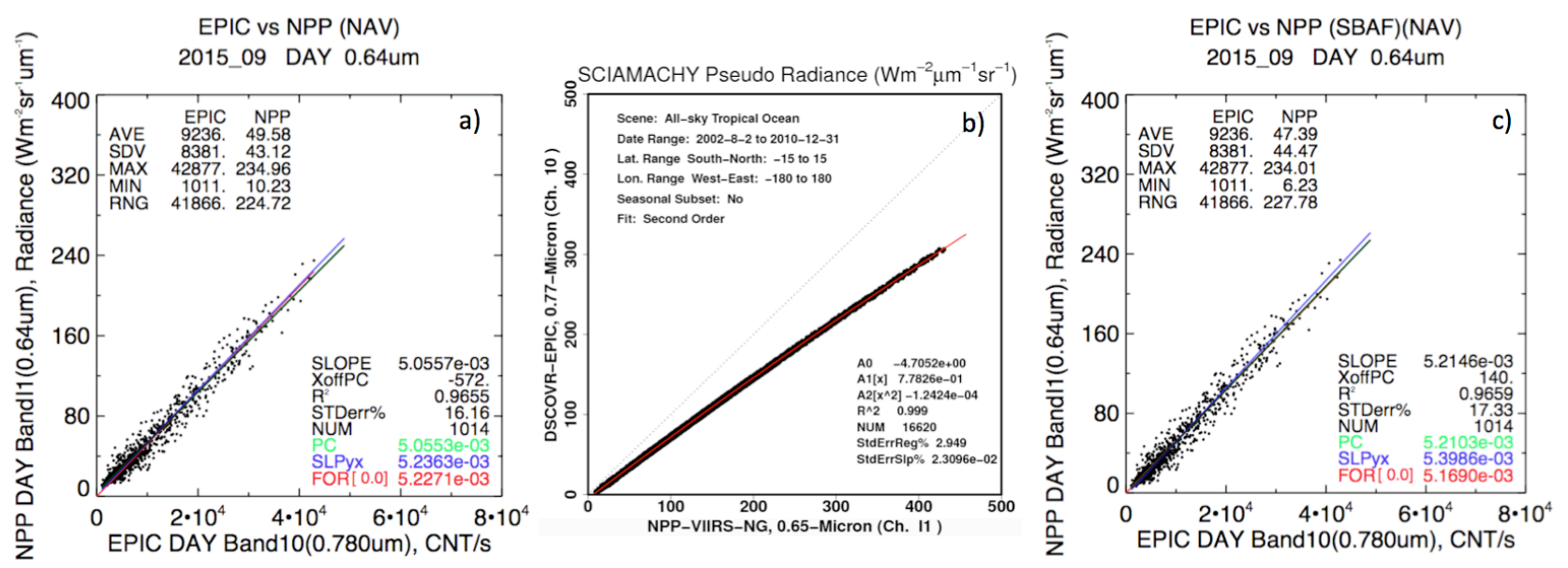

Figure 5. a) E10/I1 regression with no SBAF application. b) $2^{\text {nd }}$-order polynomial SBAF plot for E10/I1. c) E10/I1 regression with SBAF applied. See Fig. 2 for an explanation of the plot statistics. 

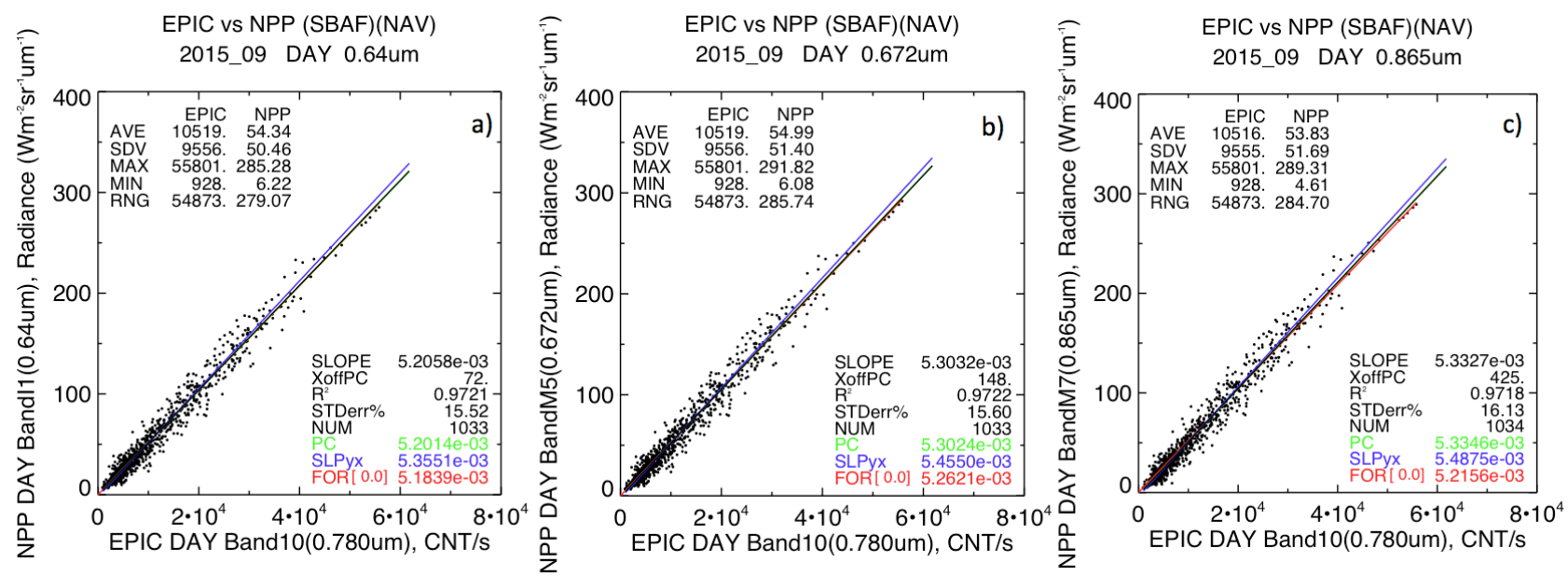

Figure 6. a) E10/I1 regression with SBAF application. b) same as a) except for E10/M5. c) same as a) except for E10/M7. See Fig. 2 for an explanation of the plot statistics.

\subsection{EPIC Temporal Gain Degradation}

In order to monitor the degradation of the EPIC instrument channels, the publicly released version 01 EPIC data were analyzed from July 2015 through June 2016. For this study, only the E5/A3, E6/A4, and E7/A1 are evaluated for temporal degradation. Both the gridded and DCC EPIC/MODIS ray-matched radiance pairs are regressed monthly. There are still possibly some varying degrees of navigation errors that may not be rectified by the navigation correction described in Section 2.1. The large amount of data also allowed application of some additional filters to ensure an optimum ratio of dynamic range, spatial uniformity, and angular matching thresholds in order to provide the greatest confidence to the regression itself. The abundance of version 01 measurements allowed the application of GAM and SVS for the gridded ray-matching technique.

Figure 7a shows the impact of applying GAM filtering discussed in Section 2.2. Note the reduction of measurements for radiances less than $200 \mathrm{Wm}^{-2} \mathrm{sr}^{-1} \mu \mathrm{m}^{-1}$. The clear-sky radiance pairs are now tightly distributed, suggesting that the clear-sky radiance pair noise was mainly caused by the angular matching differences. The monthly linear fit standard errors were mostly between $6 \%$ and $8 \%$, and the mean offset was 215 counts/second (not shown). Figure $7 \mathrm{~b}$ shows the monthly force fit gains, as a function of day since launch (DSL) of the DSCOVR satellite, from July 2015 to June 2016. The monthly force fit gains indicate no temporal degradation for E7. The gridded ray-matching gains have a $0.8 \%$ standard error about the linear temporal trend.

Figure 7c shows the corresponding DCC ray-matched radiance pairs and statistics for June 2016. The bright DCC $0.25^{\circ}$ cell radiance pairs have a standard error of $3.8 \%$ with respect to the linear fit. The average monthly standard error over the timeline was $4 \%$, which is less than the $6 \%$ to $8 \% \mathrm{SE}$ of the monthly gridded ray-matching. The DCC raymatching calibration technique ensures bright, uniform Earth scenes, for which the mean monthly A1 radiance is 380 $\mathrm{Wm}^{-2} \mathrm{sr}^{-1} \mu \mathrm{m}^{-1}$. DCC also have the lowest SBAF uncertainties. Figure $7 \mathrm{~d}$ shows the DCC monthly calibration gains and corresponding linear fit. The DCC calibration method does not show any temporal trending. The temporal linear fit standard error is $1.2 \%$, which was greater than its gridded ray-matching counterpart. The timeline mean gridded and DCC ray-matching gains differ by $1.1 \%$. The E5/A3 and E6/A4 also showed that the gridded and DCC gains differed by $1.2 \%$ and $1.1 \%$, respectively. Some of this difference maybe a result of the navigation errors that were not accurately corrected, which would effect the DCC more than gridded ray-matching given that the DCC technique uses the smaller $0.25^{\circ}$ regions. Any navigation shift between EPIC and MODIS images would also have an inaccurate angle value. Further screening and improvements in the navigation are being developed. 

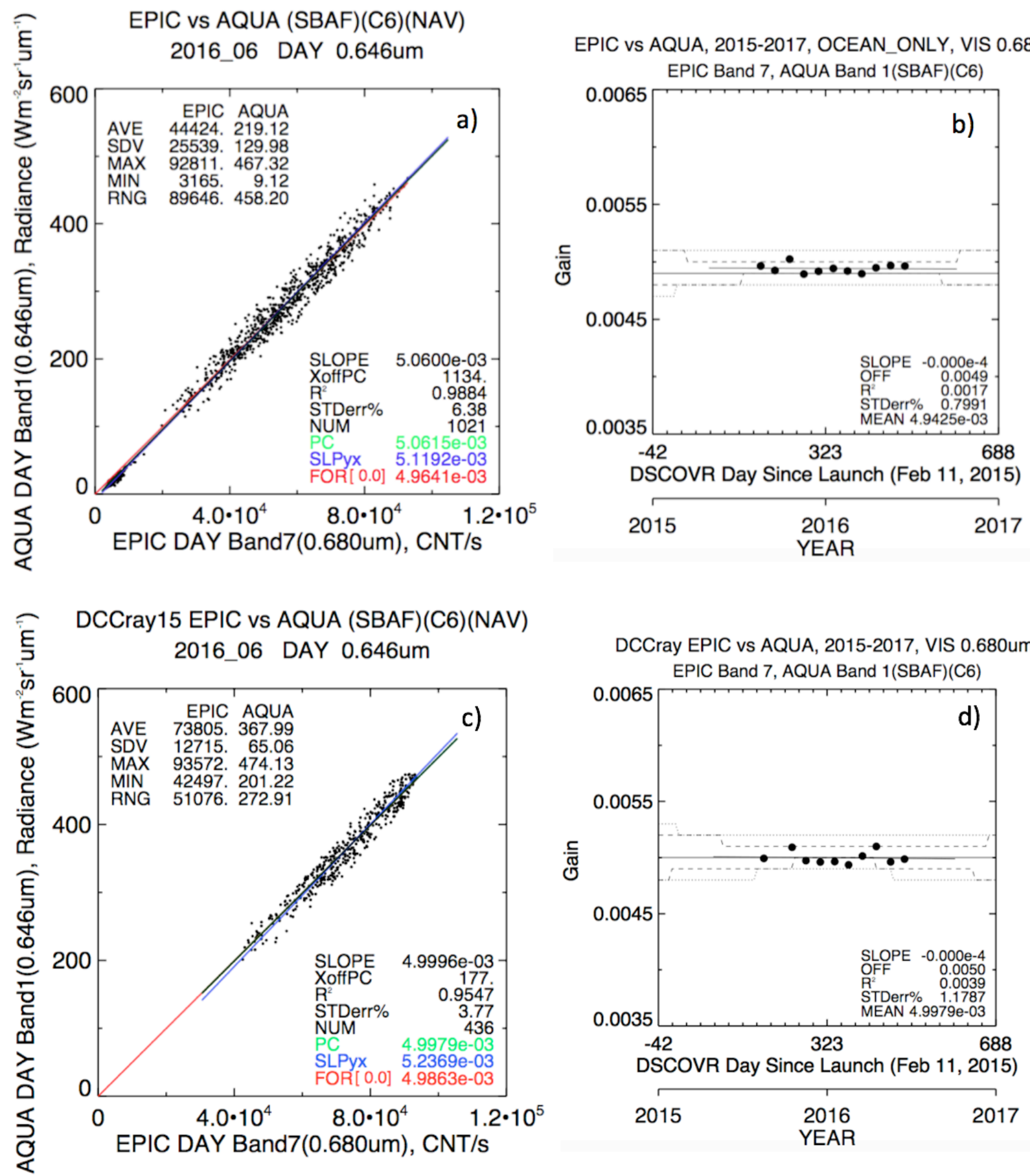

Figure 7. a) June 2016 E7/A1 for gridded ray-matching. b) Timeline of the gridded ray-matching gain. c) Same as $a$, except for DCC ray-matching. d) Same as $b$, but for DCC ray-matching. See Fig. 2 for an explanation of the plot statistics in a) and c).

\section{CONCLUSIONS}

The EPIC instrument onboard the DSCOVR satellite has a constant view of the daytime disk of Earth, and therefore provides a unique opportunity for inter-calibration of its spectral channels with well-calibrated LEO instruments, such Aqua-MODIS and NPP-VIIRS. DCC and all-sky tropical ocean gridded ray-matching were the 
techniques chosen for inter-calibration with MODIS and VIIRS. Due to the inadequacy of the EPIC navigation, an automated dynamic navigation correction method was developed to more correctly align the portion of interest of the EPIC images with that of the well-navigated LEO instruments. Results show that the navigation correction works favorably to reduce the uncertainty of the ray-matched calibration. The automated navigation correction routine is accurate within $0.25^{\circ}$, however, further navigation is required to employ invariant Earth target calibration techniques, (e.g., deserts). Most of the invariant Earth target calibration methods require near-nadir measurements and favorable orbit repeat cycles to minimize the effects of the BRDF correction ${ }^{12}$. Currently the EPIC operational sampling is limited by the transmission rate to Earth, limiting the sampling to a few times a day. Also the invariant target DCC calibration method requires pixel-level visible radiances to be collocated with IR pixels ${ }^{9}$. The feasibility of these calibration techniques will be investigated in the future.

The EPIC stray-light corrected data provided by the DSCOVR science team improved the EPIC image quality by bringing the PC fit offset closer to zero. This correction allowed a more linear response of the EPIC radiances. It is recommended that the DSCOVR science team implement this correction to the next version of data.

Not accounting for spectral band differences could bias the overall EPIC calibration gain. SCIAMACHY-based SBAFs were applied to over-lapping EPIC and VIIRS channel pairs. After applying SBAFs, all EPIC/VIIRS channel pairs showed consistent force and linear fits, and the offsets were closer to zero. This suggests that the SBAF correction brought the VIIRS radiances in line with the EPIC channel spectral response functions. The SBAF was tested with the E10 channel, which does not overlap with any of the VIIRS bands. Force fit gains were computed for E10/I1 and E10/M5 and found to be within $1.5 \%$ of one another. To determine whether this difference was due to the SBAF methodology or the VIIRS absolute calibration difference between I1 and M5 bands, force fit gains were computed for E7/I1 and E7/M5 bands. The same 1.5\% gain difference was observed, suggesting that the gain difference is due to the VIIRS I1 and M5 calibration difference, thereby validating that the SBAF algorithm can also be applied to nonoverlapping bands.

Both the all-sky ocean and DCC ray-matching techniques were employed to determine the temporal degradation of the EPIC channels based on the year of publically released version 01 data. A year of EPIC data were inter-calibrated with MODIS for E5/A3, E6/A4, and E7/A1. Neither the all-sky ocean nor DCC ray-matching techniques showed any discernable temporal degradation for the E7 band with respect to the A1 band. The all-sky ocean and DCC mean timeline gain difference was found to have a $1.1 \%$ discrepancy, which may be linked to the navigation quality.

Future work involves providing the DSCOVR science team with EPIC channel calibration gain coefficients that reference either MODIS or VIIRS, and associated temporal trends. Additionally, there is a need to work with the DSCOVR science team to validate any navigation and stray light improvements.

\section{ACKNOWLEDGEMENTS}

The DSCOVR EPIC version 01 data were obtained from the NASA Langley Research Center Atmospheric Science Data Center. The remaining EPIC navigation and stray-light corrected datasets were obtained from the DSCOVR project science team. The MODIS and VIIRS granules were obtained from the CERES project. This study was supported by NASA ROSES proposal NNH14ZDA001N-DSCOVR A.22 DSCOVR Earth Science Algorithms.

\section{REFERENCES}

[1] Doelling, D. R., A. Wu, X. Xiong, B. R. Scarino, R. Bhatt, C. O. Haney, D. Morstad, and A. Gopalan, 2015, "The radiometric stability and scaling of collection 6 Terra and Aqua-MODIS VIS, NIR, and SWIR spectral bands," IEEE Trans. Geosci. Remote Sens., Vol. 53, No. 8, 4520-4535, DOI: 10.1109/TGRS.2015.2400928.

[2] Bhatt, R., D. R. Doelling, A. Wu, X. Xiong, B. R. Scarino, C. O. Haney, and A. Gopalan, 2014, "Initial Stability Assessment of S-NPP VIIRS Reflective Solar Band Calibration using Invariant Desert and Deep Convective Cloud Targets," Remote Sens. 2014, 6, 2809-2826; doi:10.3390/rs6042809. 
[3] Minnis, P., L. Nguyen, D. R. Doelling, D. F. Young, and W. Miller, 2002, "Rapid Calibration of Operational and Research Meteorological Satellite Imagers. Part I: Evaluation of Research Satellite Visible Channels as References," $J$. Atmos. Oceanic Technol. 19, 1233-1249.

[4] Doelling, D. R., P. Minnis, and L. Nguyen, 2004, "Calibration comparisons between SEVIRI, MODIS, and GOES data," Proc. MSG RAO Workshop, Salzburg, Austria., 10-11 September, 149-154.

[5] Morstad, D. L., D. R. Doelling, R. Bhatt, and B. Scarino, 2011, "The CERES calibration strategy of the geostationary visible channels for CERES cloud and flux products," Proc. of SPIE, Earth Observing Syst. XVI, 815316.

[6] Doelling, D. R., C. O. Haney, B. R. Scarino, A. Gopalan, and R. Bhatt, 2016, "Improvements to the geostationary imager ray-matching calibration algorithm for CERES Edition 4,” submitted J. Atmos. Oceanic Technol. June 2016.

[7] Doelling, D. R., R. Bhatt, D. Morstad, 2011, "Algorithm Theoretical Basis Document (ATBD) for ray-matching technique of calibrating GEO Sensors with Aqua--MODIS for GSICS," (http://gsics.atmos.umd.edu/pub/Development/AtbdCentral/GSICS_ATBD_RayMatch_NASA_2011_09.pdf).

[8] Scarino, B. R., D. R. Doelling, P. Minnis, A. Gopalan, T. Chee, R. Bhatt, C. Lukashin, and C. O. Haney, 2016, "A web-based tool for calculating spectral band difference adjustment factors derived from SCIAMACHY hyperspectral data," IEEE Trans. Geosci. Remote Sens., vol. 54, no. 5, pp. 2529-2542.

[9] Doelling, D. R., D. Morstad, B. Scarino, R. Bhatt, and A. Gopalan, 2013, "The Characterization of Deep Convective Clouds as an Invariant Calibration Target and as a Visible Calibration Technique," IEEE Trans. Geosci. Remote Sens., 51, 1147-1159, doi: 10.1109/TGRS.2012.2225066.

[10] Mu, Q., A. Wu, T. Chang, A. Angal, D. Link, X. Xiong, D. R. Doelling, R. Bhatt, 2016, “Assessment of MODIS On-orbit Calibration using a Deep Convective Cloud Technique," SPIE this issue.

[11] G. Hong, G. Heygster, J. Notholt, and S. A. Buehler, "Interannual to diurnal variations in tropical and subtropical deep convective clouds and convective overshooting from seven years of AMSU-B measurements," J. Clim., vol. 21 , no. 17, pp. 4168-4189, Sep. 2008.

[12] Bhatt, R., D. R. Doelling, D. Morstad, B. Scarino, and A. Gopalan, 2014, "Desert-based absolute calibration of successive geostationary visible sensors using a daily exoatmospheric radiance model," IEEE Trans. Geosci. Remote Sens., 52, 3670-3682, doi: 10.1109/TGRS.2013.2274594. 